Presented at: VII Workshop on "Advanced

Accelerator Concepts

Lake Tahoe, CA

CONF $-9610210--6$

October 13-18, 1996

BNL- 63584

Micro-bunching Diagnostics for the IFEL by Coherent Transition Radiation

Y. Liu and D. B. Cline

Center for Advanced Accelerators, University of California, Los Angeles, CA 90095

X. J. Wang, M. Babzien, J. M. Fang, and V. Yakimenko

Brookhaven National Laboratory, Upton, NY 11973

RECEVER

DEC 231996

OSTI

October 1996

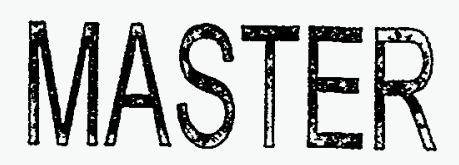

National Synchrotron Light Source

Brookhaven National Laboratory

Upton, NY 11973

Work performed under the auspices of the U.S. Department of Energy, under contract DE-AC02-76CH00016 


\section{DISCLAIMER}

This report was prepared as an account of work sponsored by an agency of the United States Government. Neither the United States Government nor any agency thereof, nor any of their employees, makes any warranty, express or implied, or assumes any legal liability or responsibility for the accuracy, completeness, or usefulness of any information, apparatus, product, or process disclosed, or represents that its use would not infringe privately owned rights. Reference herein to any specific commercial product, process, or service by trade name, trademark, manufacturer, or otherwise does not necessarily constitute or imply its endorsement, recommendation, or favoring by the United States Government or any agency thereof. The views and opinions of authors expressed herein do not necessarily state or reflect those of the United States Government or any agency thereof. 


\section{DISCLAIMER}

Portions of this document may be illegible in electronic image products. Images are produced from the best available original document. 


\title{
Micro-bunching Diagnostics for the IFEL by Coherent Transition Radiation
}

\author{
Y. Liu and D.B. Cline \\ Center for Advanced Accelerators, \\ University of California, Los Angeles, CA 90095 \\ X.J. Wang, M. Babzien, J.M. Fang and V. Yakimenko
}

Brookhaven National Laboratory, Upton, NY 11973

\begin{abstract}
Here, we propose an effective method for detecting micro-bunching effects (10-fs bunch length) produced by the IFEL interaction, by measuring the CTR spectrum. The pre-bunching of an initially energy-modulated $e^{-}$beam passing through a wiggler (IFEL interaction) is studied. Simulation shows that more than $40 \%$ of electrons are pre-bunched in the micro-bunches. The longitudinal distribution of an optically pre-bunched beam is Fourier analyzed to find the dominant harmonics contributing to the CTR. The CTR spectrum is calculated analytically for the IFEL situation. A detection system has been built to demonstrate this technique.
\end{abstract}

\section{INTRODUCTION}

The Inverse Free Electron Laser (IFEL) acceleration ${ }^{1,2}$ has been observed at the Accelerator Test Facility (ATF) at Brookhaven National Laboratory. Recently it was decided to study the bunching by the IFEL for the possible use as an injector for a high energy Inverse Cerenkov Accelerator (ICA). ${ }^{3}$ Since the IFEL operates in a vacumm environment, it is possible to provide a high quality, pre-bunched $\epsilon^{-}$beam for the next acceleration stage. As we know, ICA operates in a phase matching medium with diamond windows which are used to isolate the medium from the vacumm environment. These additional materials will scatter electrons and change $e^{-}$beam emittance. 
A basic approach for such a two-stage system is to send an $e^{-}$beam into a laser-driven pre-buncher (IFEL wiggler), whose output feeds directly into the laser accelerator (ICA gas cell). The IFEL stage is needed to maximize the number of electrons maintaining a specific phase relationship with the optical mode required for optimum acceleration. An optical mirror will be mounted in the middle of the ICA gas cell which could be inserted into the interaction region by remote control. It is a diagnostic element using Coherent Transition Radiation (CTR) to monitor micro-bunchingin a planned IFEL pre-buncher, ICA accelerator experiment.

We have previously studied the use of a CTR to predict the bunching of the ICA. ${ }^{4}$ In this paper the IFEL self-bunching process (without the space-charge effect) has been simulated. Photon production from the CTR is estimated according to the current IFEL setup at the ATF. Furthermore, a feasible CTR diagnostic system to detect and measure the micro bunches is presented.

\section{OPTIMUM SELF-BUNCHING DISTANCE AND ELECTRON DISTRIBUTION}

The $\epsilon^{-}$beam passing through the wiggler and interacting with the high intensity $\mathrm{CO}_{2}$ laser leaves the interaction region with a distinct velocity distribution pattern which, propagated over a certain distance, results in self-bunching of the $e^{-}$beam. In our bunching experiment, this characteristic distance is optimized to be about $45-50 \mathrm{~cm}$, and we expect to observe strong bunching on a scale of several microns. Since there is no external electromagnetic field present in the drift space, the effects of the space charge within the $\epsilon^{-}$beam will dominate and they should be considered. However, previous study using PARMELA to simulate phase-space evolution in the drift space for ICA shows that particles at the head of the bunch tend to gain energy, while particles from the tail are decelerated. ${ }^{4}$ In effect, the energy-phase space is rotated in the counter-clockwise direction. The amount of this local rotation is weighted by the longitudinal charge distribution gradient. Therefore, the strongest effect occurs near the center of the distribution (smearing). Under strong spacecharge conditions (1-nC bunch) the effect of self-bunching is still present. The bunching peak is slightly 'washed out' by the space-charge defocusing and smearing - at about the $10 \%$ level (FWHM) comparied with the case of no space charge.

In the current setup the maximum charge delivered to IFEL wiggler at ATF is up to severalhundred pC. It is far less than our simulation charges. Thus, we should not worry about space-charge effect too much in the IFEL self-bunching study. By optimizing laser power and magnetic field at $40 \mathrm{MeV} e^{-}$beam energy we predict that more than $40 \%$ of electrons are bunched within several microns at the optimum bunching distance. Figure 1b shows the electron distribution in the longitudinal direction at predicted optimum bunching distance. Figure la shows the electron distribution in energy-phase space. The code we used to simulate the electron energy modulation is a 1D IFEL Fortran code written by J. Gallardo at BNL. 5 

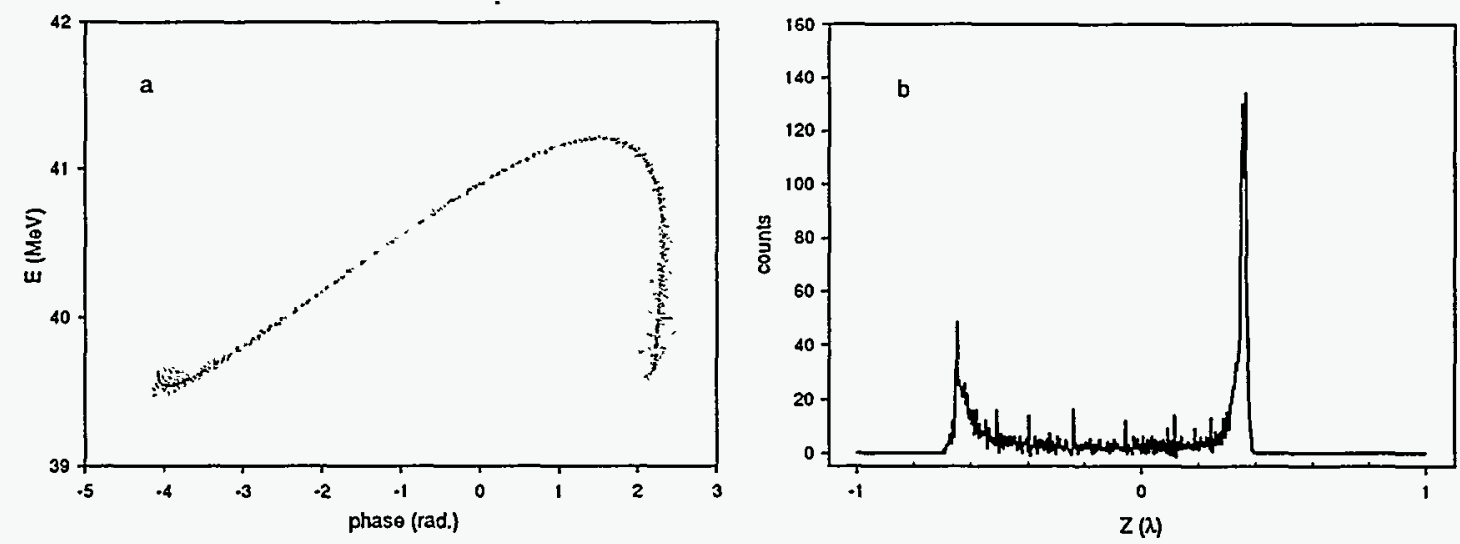

Figure 1: (a) The electron distribution at predicted optimum bunching distance $(50 \mathrm{~cm})$ in energy-phase space. $\lambda=10.6 \mu m$ (b) The electron distribution in real space.

\section{COHERENT TRANSITION RADIATION}

Backward transition radiation generated by a particle crossing the interface of the two media at an oblique angle, $\theta$, is characterized by the following distribution: ${ }^{6}$

$$
\frac{d^{2} u}{d \omega d \Omega}=\frac{e^{2}}{4 \pi^{2} c} \frac{|r /|^{2} \sin ^{2} \theta}{(1-\beta \cos \theta)^{2}},
$$

where $r$ is the Fresnel reflection coefficient for the mirror. However, CTR is a collective effect produced by a large ensemble of electrons being in phase with each other. The total number of photons radiated is highly enhanced and the total radiation distribution becomes ${ }^{7}$

$$
\frac{d^{2} U}{d \omega d \Omega}=[N+N(N-1) F(\omega, \theta)] \frac{d^{2} u}{d \omega d \Omega}
$$

where

$$
F(\omega, \theta)=|f(\omega, \theta)|^{2}=\left|\iiint f(r, z) \exp (-i \vec{k} \cdot \vec{x}) d^{3} x\right|^{2}
$$

is a bunching factor, containing information about the electron distribution. The coherent effect scales like $N^{2}$ compare to the incoherent part, which scales linearly with the electron number, $N$. Since $N$ is about $10^{9}$ at least in our case, therefore Eq.(2) is simplified as

$$
\frac{d^{2} U}{d \omega d \Omega} \approx N^{2} F(\omega, \theta) \frac{d^{2} u}{d \omega d \Omega}
$$

This technique has been used at several synchrotron light sources to measure a single bunch. ${ }^{8,9,10}$ The multiple bunch case for fundamental wavelength (first harmonic wavelength) also has been estimated. 4,11 
In multiple bunches the total coherent intensity depends on electron distribution in each bunch and number of bunches. To estimate the backward CTR coming from a $45^{\circ}$ mirror for the IFEL experiment, we assume the following set of conditions describing the experimental setup at the ATF. (1) The initial $e^{-}$beam distribution (before entering the IFEL wiggler, where the electrons are modulated by the $\mathrm{CO}_{2}$ laser) is assumed to be a smooth bi-Gaussian in $r$ and $z$. (2) The energy modulation imposed in the interaction region is strong enough and the affected electrons should self-bunch at a certain distance outside the wiggler. Under such assumptions we should expect that the modulated electron distribution evolves in a drift space $(z)$ according to the following formula ${ }^{4,11}$ :

$$
\begin{aligned}
f(r, z) & =g(r) h(z) \\
& =\left[\frac{\exp \left(-r^{2} / 2 \sigma_{r}^{2}\right)}{2 \pi \sigma_{r}^{2}}\right]\left[\frac{\exp \left(-z^{2} / 2 \sigma_{z}^{2}\right)}{(2 \pi)^{1 / 2} \sigma_{z}^{2}}\left[1+\sum_{n=1}^{\infty} b_{n} \cos \left(n k_{r} z\right)\right]\right],
\end{aligned}
$$

where $\sigma_{z}$ and $\sigma_{r}$ present the longitudinal and the vertical $\mathrm{e}^{-}$beam size, $k_{r}$ is the wave-number of the $\mathrm{CO}_{2}$ laser modulation and $b_{n}$ are the Fourier coefficients of the longitudinal electron density distribution.

By combining Eq. (3) with Eq. (5) the transverse part $F_{T}$ and the longitudinal part $F_{L}$ are given by the following expressions:

$$
\begin{gathered}
F_{T}(\omega, \theta)=\left|\iint g(r) \exp (-i k r \sin \theta \cos \phi) r d r d \phi\right|^{2} \\
=\exp \left(-\left(k \sigma_{r} \sin \theta\right)^{2}\right), \\
F_{L}(\omega, \theta)=\left|\int h(z) \exp (-i k i z \cos \theta) d z\right|^{2} \\
=\mid \exp \left(-\frac{\left(k \sigma_{z} \cos \theta\right)^{2}}{2}\right)+\sum_{n=1}^{\infty}\left(\frac{b_{n}}{2}\right)\left[\exp \left(-\frac{\sigma_{z}^{2}}{2}\left[k \cdot \cos \theta-n k_{r}\right]^{2}\right)\right. \\
\left.+\exp \left(-\frac{\sigma_{z}^{2}}{2}\left[k \cos \theta+n k_{r}\right]^{2}\right)\right]\left.\right|^{2} .
\end{gathered}
$$

Since the micro-bunch peaks are narrow compared to their separation distance $\left(k_{r} \sigma_{z} \gg 1\right)$, only the contributions from near each harmonic wavelength could be significant. Thus, we can simplify Eq. (7) referring to each harmonic wavelength as follows:

$$
F_{L}(\omega, \theta) \approx\left(\frac{b_{n}}{2}\right)^{2}\left[\exp \left(-\sigma_{\tilde{z}}^{2}\left(k \cos \theta-n k_{r}\right)^{2}\right)\right] .
$$

The total number of the CTR photons in the narrow band around the harmonic wavelength is given by the following expression:

$$
\frac{d^{2} N_{p l}}{d k d \theta} \approx \frac{\alpha|r|^{2}}{2 \pi}\left(\frac{N b_{n}}{2}\right)^{2} \frac{\sin ^{3} \theta}{(1-\beta \cos \theta)^{2}} \frac{1}{k} \exp \left[-\left(k \sigma_{r} \sin \theta\right)^{2}-\sigma_{z}^{2}\left(k \cos \theta-n k_{r}\right)^{2}\right]
$$


where $\alpha=e^{2} / \hbar c$. To find the angular distribution of the CTR photons in the narrow band (approximately one percent bandwidth) around this frequency, we should integrate Eq. (9) over the described frequency range. Due to a very narrow frequency band, the value of $k$, in the fourth term of Eq. (9), could be set to $n k_{r}$ and then the integration limits could be exterided from $-\infty$ to $\infty$. Finally, we get the angular distribution of the CTR photons around each harmonic frequency, in the following compact form:

$$
\begin{aligned}
\frac{d N_{p /}}{d \theta} \approx & \frac{\alpha\left|r^{\prime}\right|^{2}}{2 \pi}\left(\frac{N b_{n}}{2}\right)^{2} \frac{1}{k_{r} \sigma_{z}} \frac{\sin ^{3} \theta}{(1-\beta \cos \theta)^{2}}\left(\frac{1}{n}\right) \times \\
& {\left[\frac{\pi}{\left(\left(\sigma_{r} / \sigma_{z}\right) \sin \theta\right)^{2}+\cos ^{2} \theta}\right]^{1 / 2} \exp \left[-\frac{\left(n k_{r} \sigma_{r} \sin \theta\right)^{2}}{\left(\left(\sigma_{r} / \sigma_{z}\right) \sin \theta\right)^{2}+\cos ^{2} \theta}\right] . }
\end{aligned}
$$

In general $\sigma_{r} \ll \sigma_{z}$ and $\theta \ll 1$. Using this approximation, Eq. (10) reduces to the following simple formula:

$$
\frac{d N_{p h}}{d \theta} \approx \frac{\alpha|r /|^{2}}{8 \sqrt{\pi^{3}}}\left(\frac{k_{r} \sigma_{z}}{\pi}\right)\left(\pi \frac{N b_{n}}{k_{r} \sigma_{z}}\right)^{2}\left(\frac{1}{n}\right) \frac{\sin ^{3} \theta}{(1-\beta \cos \theta)^{2}} \exp \left[-\left(n k_{r} \sigma_{r} \sin \theta\right)^{2}\right] .
$$

The physical meaning of Eq. (11) is quite clear. The second term represents the full length of the macro-bunch in units of the modulation wavelength $\lambda_{r}$. The third term is the square of the total number of electrons within the micro-bunch (the laser modulation 'chops' the macro-bunch into a number of micro-bunches). The fourth term describes the contribution of the single-electron transition radiation and the last term reflects the transverse distribution of electrons. $n$ is a harmonic number. The coherent contribution from higher harmonic will be significantly weaker than fundamental wavelength. By Fourier analysis of the CTR spectrum at the optimum bunching distance, the coefficients $b_{n}$ are easily found. Since the micro-bunches are very narrow the contribution from the higher harmonic components may be detectable in our experiment. Here, we estimate the number of photons generated by each harmonic, separately.

For the IFEL experimental conditions, a $40-\mathrm{MeV} e^{-}$beam pulse $\left(2 \sigma_{z} \sim 3000 \mu \mathrm{m}\right)$ is about $10 \mathrm{ps}$ long. Its $\sigma_{r}$ at the focal waist is about $300 \mu \mathrm{m}$. After passing through $50 \mathrm{~cm}$ of the drift space the beam size will grow and reach the transverse size given by $\sigma_{r} \sim 390 \mu \mathrm{m}$. The total number of electrons in a single macro-bunch is assumed to be $N \sim 1.25 \times 10^{9}$, which is equivalent to $200 \mathrm{pC}$ of total charge. From the spectrum analysis, the Fourier coefficients are evaluated as follows: $b_{1}=1.134, b_{2}=0.978, b_{3}=0.867$ and $b_{4}=0.778$. Since the total photons at a certain wavelength will significantly decrease with increasing harmonic number (see Eq. (11)), therefore we confine our consideration to the first four harmonics only. The total number of photons at these wavelengths is evaluated as follows: $N_{1} \sim 5.90 \times 10^{11}$, $N_{2} \sim 1.79 \times 10^{10}, N_{3} \sim 1.97 \times 10^{9}$ and $N_{4} \sim 3.86 \times 10^{8}$. These values imply that the IFEL CTR signal is quite strong. The possibility of detecting the first, second, even third or fourth harmonic frequencies with a narrow bandwidth detector gives more flexibility to our measurement. Theoretically predicted angular distributions for the first four harmonics are shown in Figure 2. As a comparison, incoherent transition radiation distribution is shown in Figure 2 also. 


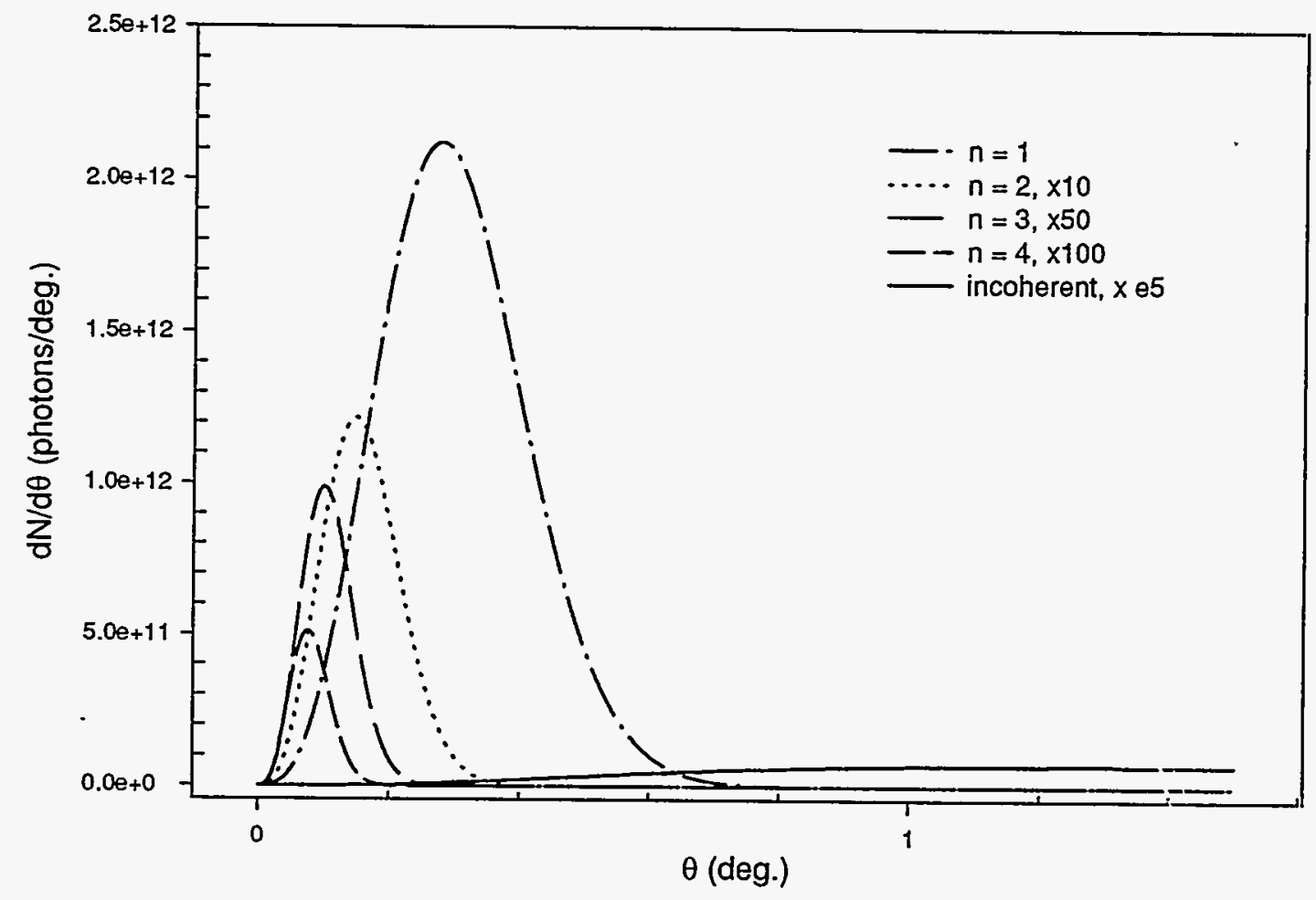

Figure 2: Angular distribution of the CTR photons within $1 \%$ bandwidth. (a) $n=1$, first harmonic frequency, peak at $\theta_{m}=0.28^{\circ}$; (b) $\mathrm{n}=2$, second harmonic frequency, peak at $\theta_{m}=0.15^{\circ}$; (c) $\mathrm{n}=3$, third harmonic frequency, $\theta_{m}=0.10^{\circ} ;$ (d) $\mathrm{n}=4$, fourth harmonic frequency, $\theta_{m}=0.06^{\circ}$; and (e) incoherent, $\theta_{m}=1.27^{\circ}$.

\section{DIAGNOSTIC SETUP FOR THE IFEL}

At the ATF, beam-line 2 is provided for the IFEL experiment. The detection system has been assembled and is located downstream near the wiggler exit. The basic experimental setup can be described as follows. A $45^{\circ}$ mirror (CTR mirror) held by a 6-way cross is inserted in the $e^{-}$beam path to generate backward CTR and transport the CTR light through a ZnSe window into the detecting system. The CTR light will be focused by a 3" diameter remote-controlled parabolic mirror into a cooled detector (InSb) with $1 \times 1 \mathrm{~mm}^{2}$ sensitive area. The CTR mirror can be moved in and out easily by an actuator. The 6-way cross connects with a pair of bellows which allow the mirror to travel back and forth for $40 \mathrm{~cm}$ along the $e^{-}$beam axis without disturbing the vacumm environment. The whole system including the 6-way cross sits on a movable table driven by a digitized remote-controlled stepping motor. A strip-line detector is placed between the upstream bellows and the exit of 
wiggler to provide the $e^{-}$beam charge information. The schematic of the complete detection system is shown in Figure 3. Control and data collection will be performed at the ATF control room.

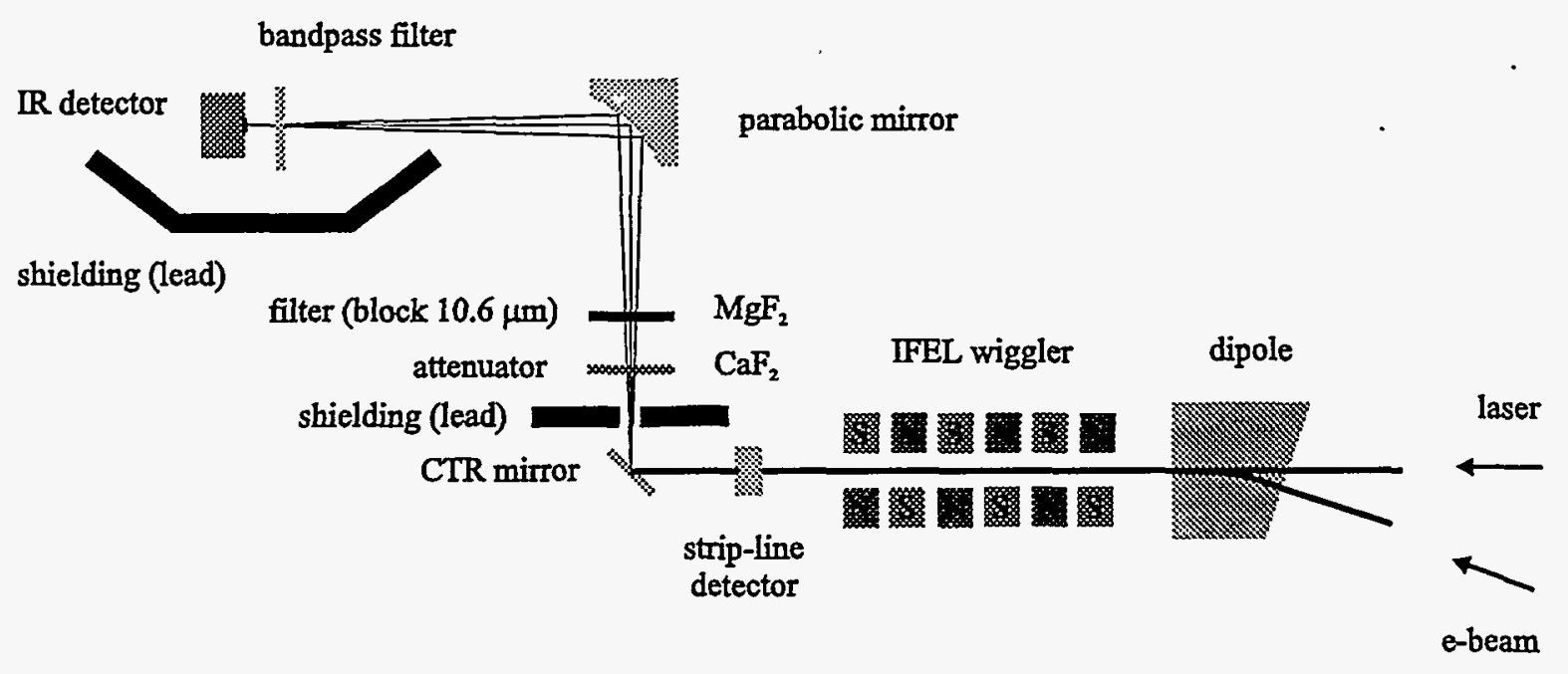

Figure 3: Schematic of the CTR diagnostic setup for the IFEL experiment.

The disadvantage of measuring the backward CTR is the fact that the intensity may be slightly weaker than for the forward CTR, diminished by the factor $|r \prime|^{2}$. Due to IFEL setup $\mathrm{CO}_{2}$ laser will directly hit the CTR mirror and be transported into the detection system. This implies that the first harmonic $(10.6 \mu \mathrm{m})$ CTR will not be useful to identify bunching effect. A filter should be placed between $\mathrm{ZnSe}$ window and parabolic mirror to block the $\mathrm{CO}_{2}$ laser light. Only second and higher harmonic frequencies CTR should be detected. In principle it is not necessary to use the filter since the detector is not sensitive to this wavelength. But the laser intensity is too strong without attenuation, and damage the detector.

By moving the CTR mirror back and forth the optimum bunching distance could be located by looking at the micro-bunching effect variation. The adjustment of the input laser power at the fixed $e^{-}$beam energy and magnetic field is an alternative way of finding the optimum bunching distance. However, it may affect the bunching characteristics significantly, which will lead to a complex calibration procedure.

In conclusion, this is a flexible and easily operated detection system. It could help us optimize phase matching condition quickly in planned IFEL/ICA joint experiment. The detection system has been built to demonstrate this technique.

\section{ACKNOWLEDGMENTS}

This work was supported by the U.S. Department of Energy, Grant No. DE-FG0392ER40695. We thank I. Ben-Zvi, E.B. Blum, J. Gallardo, W. Kimura, I. Pogorelsky, J. 
Sandweiss and A. van Steenbergen for their help and discussions. Also we thank J. Skaritka for the engineering design work and B. Cahill, B. Harrington, M. Montemagno and K. Kusche for their technical support.

\section{REFERENCES}

1. A. van Steenbergen, J. Gallardo, J. Sandweiss, J.M. Fang, M. Babzien, K. Batchelor, A. Fisher, K. Kusche, R. Malone, I. Pogorelsky, J. Qiu, T. Romoano, J. Sheehan, J. Skaritka, T. SrinivasanRao, X.J. Wang, "Inverse Free Electron Laser $e^{-}$Acceleration", Proc. BNL/ATF Users Meeting, December, 1995

2. A. van Steenbergen, J. Gallardo, J. Sandweiss, J.M. Fang, M. Babzien, J. Qiu, J. Skaritka and X.J. Wang,"Observation of Energy Gain at the BNL Inverse Free Electron Laser Accelerator", to be pub., PRL

3. W.D. Kimura, et al, Phys. Rev. Lett. 74(4), 546 (1995).

4. Y. Liu, et al., AIP Conference Proceedings 367, AIP Press, New York, 445.

5. Private communication.

6. D.W. Rule, et al, Nucl. Instr. Methods in Phys. Res. A296, 739 (1990).

7. E.B. Blum, U. Happek and A.J. Sievers, Nucl. Instr. Methods in Phys. Res. A307, 568 (1991).

8. T. Nakazato, et al, Phys. Rev. Lett. 63(12), 1245 (1989).

9. Y. Shibata, et al., Phys. Rev. E, 50(2), 1479 (1994).

10. R. Lai and A.J. Sievers, Phys. Rev. E, 50(5), R3342 (1994).

11. J. Rosenzweig, G. Travish, and A. Tremaine, Nucl. Instr. Methods in Phys. Res. A365, 255 (1995). 